\title{
A LITERATURE REVIEW ON SUPPORT FOR EATING HABITS OF PATIENTS WITH CANCER IN JAPAN
}

\author{
Keiko Sekido 1), Kiyomi Harada 1), Kaori Tsutsumi 2), \\ Sayoko Uemura 3), Hitomi Takeichi 4) and Hiroto Sekido 5)
}

\begin{abstract}
1) Kyoto Prefectural University of Medicine, School of Nursing, Kyoto, Japan. 2) Sonoda Women's University, Department of Human Nursing, Hyogo, Japan.

3) Bukkyo University, Department of nursing, Kyoto, Japan.

4) Kyoto Kacho University, Department of Food and Nutrition, Kyoto, Japan.

5) Kyoto University, Institute for Liberal Arts and Sciences, Kyoto, Japan
\end{abstract}

\section{Introduction}

Objectives

\section{Results}

Cancer treatment is often accompanied by adverse effects that are unbearable to patients with cancer and may exacerbate a deterioration in their nutritional status. It is important to provide support for their eating habits so that cancer patients can ingest meals.

We conducted a literature review with the objective of clarifying what types of studies and reports have been published so far in Japan on the theme of providing support to cancer patients to improve their eating habits.

The keywords of " "patients with cancer" and "eating habits" were used in a search within Japan Medical Abstracts Society (Igaku Chuo Zasshi) website, a database website listing medical documents. Documents published during the period from 2008 to 2017 were searched. The search took place in January, 2018. In handling the literature, the author paid utmost consideration not to infringe the copyright.

From the search on the website of Igaku Chuo Zasshi, 59 documents were extracted. After excluding those documents which did not accord with the aim of this research, 25 documents remained as the subject documents for this research. The contents of the studies were classified into "special dietary conditions for patients with cancer(13)", "meals for patients with cancer in the terminal phase(1)", "associations between sarcopenia and meals in patients with cancer(1) ", "actual conditions of the amount of dietary intake of patients with cancer(8) " and "effects of interventions by a nutritional support team(2)".

( ) =number of papers

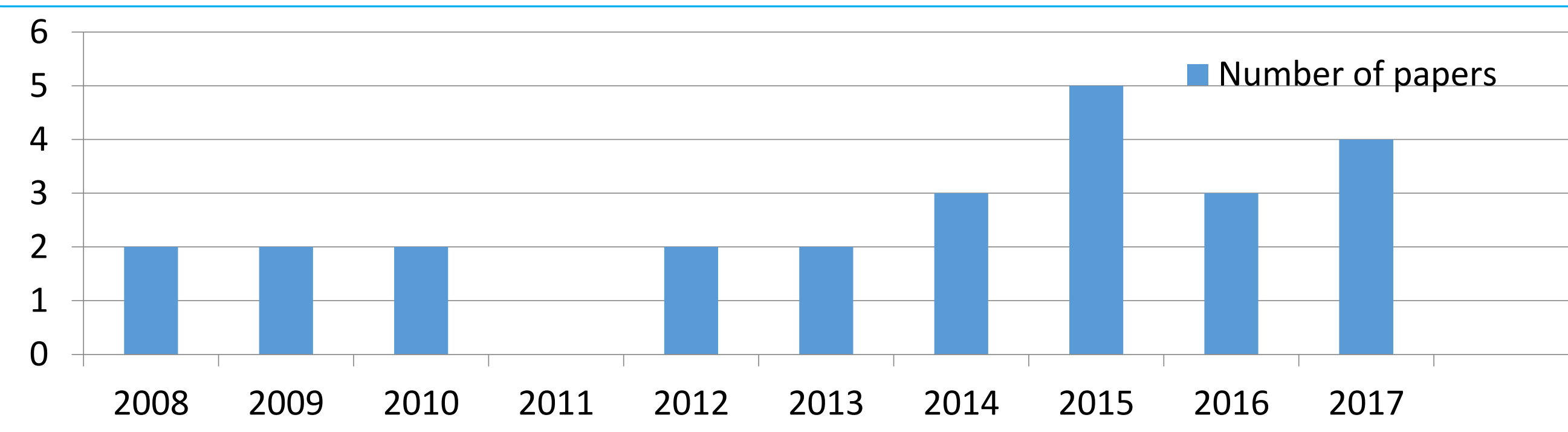

Fig. 1 The number of the extracted literature according to the publication year

Of these classifications, the largest portion of the contents of the studies was classified into "special dietary conditions for cancer patients," with the result that the amount of dietary intake by cancer patients increased following the introduction of special conditions. Each report attempted to give a range of special conditions so that meals would look beautiful, taste better for patients and might encourage them to feel like eating a meal. Many reports created special conditions by using traditional Japanese foods and flavors and placed an emphasis on tastes that the patients were familiar with. Furthermore, some reports proposed meals that used seasonal ingredients or meals that were served on a special occasion such as a festival.

Our finding suggested that meals contributed not only to the improvement of the nutritional status of cancer patients but also to the provision of enjoyment of life and enhancement of their quality of life (QOL). 